\title{
A Novel BINNINg AND INDEXING APPROACH Using HaNd Geometry ANd Palm Print To ENHANCE Performance Of Biometric IDENTIFICATION SYSTEM
}

\author{
Anitha M L and Dr RadhaKrishna Rao K A \\ P.E.S .College Of Engineering,Mandya, Karnataka, India
}

\begin{abstract}
This paper proposes a Biometric identification system for person identification using two biometric traits hand geometry and palm print. The hand image captured from digital camera is preprocessed to identify key points on palm region of hand. Identified key points are used to find hand geometry feature and palm print Region of interest (ROI). The discriminative palm print features are extracted by applying local binary descriptor on palm print ROI. In a biometric identification system the identity corresponding to the input image (probe) is determined by comparing probe template with the templates of all identities enrolled in biometric system (gallery). Response time to establish the identity of an individual increases in proportion to the number of enrollees. One way to reduce the response time is to retrieve a smaller set of candidate identity templates from the database for explicit comparison. In this paper we propose a coarseto-fine hierarchical approach to retrieve a smaller set of candidate identities called as candidate set to reduce the response time. The proposed approach is tested on the database collected at our institute. Proposed approach is of significance since hand geometry and palm print features can be extracted from the palm region of the hand. Also performance of identification system is enhanced by reducing the response time without compromising the identification accuracy.
\end{abstract}

\section{KEYWORDS}

Palm print, Hand geometry, Identification, Binning, Indexing.

\section{INTRODUCTION}

With the increasing emphasis on security, developing Computer aided person identification system is becoming increasingly important in our present day information society. Tradition identification approaches such as what a person knows or what a person has are not sufficiently reliable to satisfy the requirements of identification systems which may be fake or cracked. To meet the requirements of identification systems, Biometric systems has emerged as new type solutions to our society's ever increasing demand of improved security requirements. Applications such as passenger control in airports, access control in restricted areas, border control, database access and financial services are some of the examples where the biometric technology has been applied. The techniques for identifying an individual based on his/her physiological or behavioural characteristics are called as Biometrics [1-3]. Common physiological biometrics includes finger print, face, iris, ear, hand vein, hand geometry and palm print traits. Behavioural biometrics includes signature, gait, voice and hand writing traits. Biometric system consists of two subsystems, one for enrolment and second one for recognition. In the enrolment stage, biometric data are acquired from the individuals, feature sets are extracted 
from the acquired data, and one or multiple templates per individual are computed and stored in the database. In the recognition stage, biometric data of an individual is captured, feature set is computed (query template) and then it is compared with the templates in the database created during enrolment. Biometric systems can operate in two modes, Verification and Identification. Verification refers to confirming or denying a person's claimed identity. In this mode the system performs one to one comparisons of the query template with the individual's own biometric templates stored in the database. Identification refers to establishing a person's identity. In this mode, query template is compared with the templates of all persons enrolled in the database to establish an individual's identity. With the increase in the size of the biometric database these comparisons not only increases the time required to declare an individual's identity but also the identification error rate. Hence there is a need for new approaches for retrieving relevant candidate identities from the database against which comparison are performed to identify an individual. The retrieval of a small number of identities from database based on probe template is known as database filtering which can be achieved by classification or indexing schemes. Therefore new representation schemes that allow for faster search and shorter retrieval time are needed.

Biometric systems based on single biometric trait are referred to as unimodal systems. A single trait of an individual can sometimes be insufficient for identification due to few reasons like noise in acquired image, non universality and spoofing. For these reasons multimodal biometric systems i.e., systems that integrate two or more biometric traits are being developed to overcome the disadvantages of unimodal systems particularly to increase the recognition accuracy and to decrease the possibility of circumventing the system. In contrast to biometric traits like face and iris hand related traits such as palm print, hand geometry and inner knuckle print are attracting considerable attention in biometric research community since hand image acquisition can be done with reduced complexity set up. In this type of set up user need not hold any pegs or touch any peripheral for their hand images to be acquired. Such a set up is believed to satisfy public's demand for non invasive and hygienic biometric technology. Since palm print and hand geometry features can be extracted from a single image, in our work we have selected these two modalities for developing a novel biometric identification system for performance enhancement.

Rest of the paper is organized as follows. Section 2 presents a brief review of research in palm print and hand geometry. Section 3 describes the proposed approach. Performance of the proposed scheme is presented in section 4 followed by the last section that concludes the presented work.

\section{LITERATURE SURVEY}

Work that appears in literature with respect to palm print feature extraction are mainly lines based [4-6], sub space [7-11 ] and transform based approaches [12-15]. Line based approaches use either existing edge detection methods or develop edge detectors to extract palm lines which are matched directly or represented in other formats for matching two palm print images to make final decision. Sub space approaches use methods like principal component analysis, independent component analysis and liner discriminative analysis in which subspace coefficients are regarded as features. Distance based measures are used for comparing the features. Transform based approaches utilizes statistical methods to transform images into other domain. Gabor wavelets and Fourier transforms are the most commonly used approaches. Other approaches[16-18] combine many image processing techniques and use some standard classifiers to make final decision of recognition. Hand geometry features generally used are finger length and widths, palm length and width, area/size of palm [19-22]. 
Existing research on palm print and hand geometry is focused mainly on feature extraction and not on hand geometry feature selection. The main aim of this work is to identify a hand geometry feature that reduces search space by retrieving relevant candidate identities from the database against which comparisons are performed to identify an individual. Biometrics systems that use combination of hand traits have been proposed by various researchers. But these systems mainly address the accuracy issues of biometric systems neglecting the scalability and identification time issues of large databases. Hand geometry features are not descriptive enough for identification of individuals in large database. Whereas palm print features are reliable since they can serve as unique biometric identifier as reported in biometric literature. Based on the above mentioned fact in the proposed approach the gallery set database is partitioned into several bins using hand geometry feature of enrolees. During identification, probe template hand shape feature will be used to identify to which bin the probe template will match. Final identification is then conducted in the reduced search space using palm print modality that has a higher matching accuracy. Very few researches have studied the use of indexing unimodal biometric database to reduce the search space so that the matching phase deals with only a subset of entire database. Hence reduction of search space in biometric databases still remains as a challenging problem.

\section{Proposed Hierarchical Approach}

Figure 1 illustrates the hierarchical frame work of the proposed system. The proposed system works in two stages. First stage consists of image acquisition, pre processing and features extraction components. In the pre-processing stage the alignment and orientation of the hand images are corrected and key points on palm region are found for use in the successive stages. In feature extraction stage the most discriminating features from the hand are extracted. Second stage consists of proposed hierarchical approach and identification strategy. The details of each stage are discussed in the subsequent sections.

\subsection{Image Acquisition and Pre processing}

The image acquisition module uses digital camera, tripod for image stability and well defined background. This set up provides a simple, noncontact, comfortable and user friendly acquisition. Our data collection process spanned over one month and 448 persons volunteered for the database. 


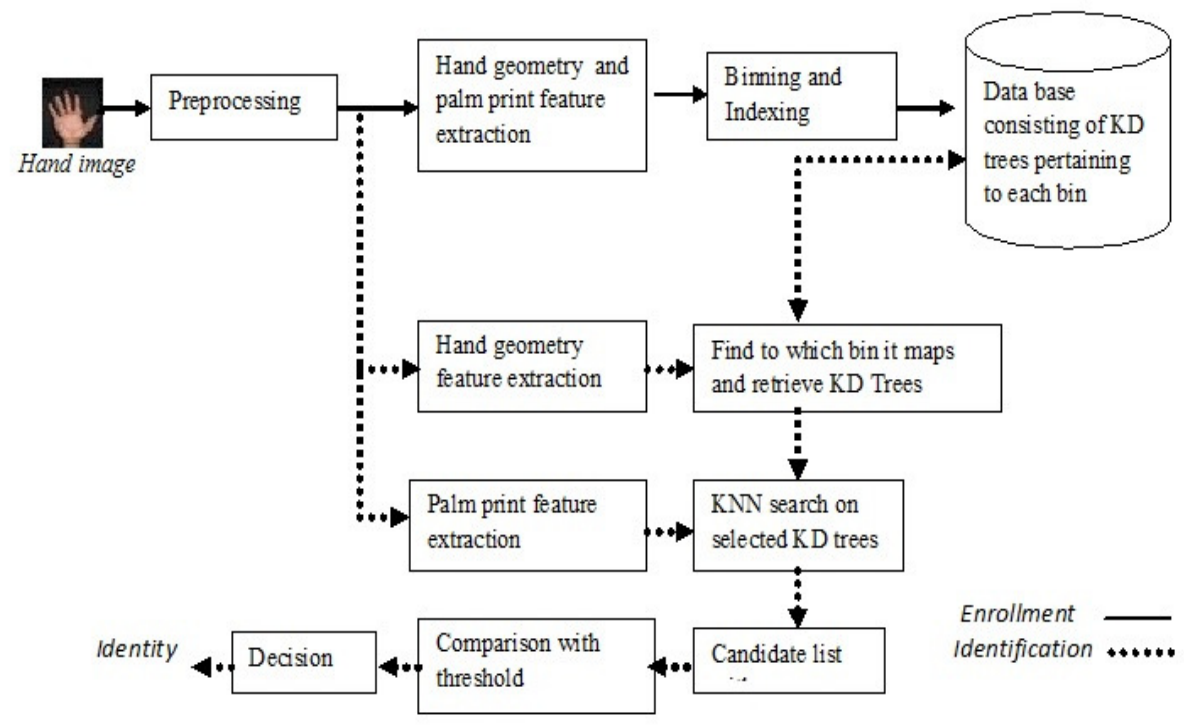

Figure 1 Proposed hierarchical framework

The participants were mainly students from our institute and were in the age group of 20 to 24 years. Four hand images of each individual were captured in two sessions resulting in 8 images per individual leading to a total of 3584 images in the database. Complete image database is divided into two mutually exclusive gallery (training) and probe (test) sets. The gallery set consists of images with known identities (ID's). However, probe set consists of images whose identity is to be known. Let $\mathrm{DB}_{\mathrm{G}}=\left\{\mathrm{IG}_{1}, \mathrm{IG}_{2}, \ldots \ldots . \mathrm{IG}_{\mathrm{N}}\right\}$ be the gallery set database consisting of $\mathrm{N}$ hand images and $\mathrm{DB}_{\mathrm{P}}=\left\{\mathrm{QP}_{1}, \mathrm{QP}_{2}, \ldots \ldots . \mathrm{QP}_{\mathrm{M}}\right\}$ be the probe set database consisting of $\mathrm{M}$ hand images.

Pre processing process involves the following steps.

a) Captured colour image is converted to gray level image since gray level image is adequate for extracting the features.

b) A fixed threshold is applied to convert the gray image into a binary image.

c) Hand contour is obtained and three key points are identified as per the algorithm proposed in [23] with certain modifications.

Identified key points $\mathrm{P} 1, \mathrm{P} 2$ and $\mathrm{P} 3$ are marked as filled circles as shown in figure 2

\subsection{Feature Extraction}

\subsubsection{Hand Geometry feature extraction}

In view of reducing the dimension of feature vector key points P1, P2 and P3 identified during pre processing stage are used for feature extraction. The angle between the line joining the points $\mathrm{P} 1, \mathrm{P} 2$ and $\mathrm{P} 2, \mathrm{P} 3$ is calculated. The angle value is stored as hand geometry feature vector of one dimension. In order to check the suitability of this feature two hand images of the same person taken in two sessions were selected. The two angle values obtained are 151 and 156 degrees respectively. It can be observed from figure 3 and 4 that even though spacing between fingers are 
different there is not much difference in the angle values computed for first session and second session images.

\subsubsection{Palm Print feature extraction}

For cropping palm print ROI following steps are used.

a. Two key points are selected from the key points identified during preprocessing stage. First point is the valley point between little finger and ring finger, second point is the valley point between middle finger and index finger. These points are considered as anchor points.

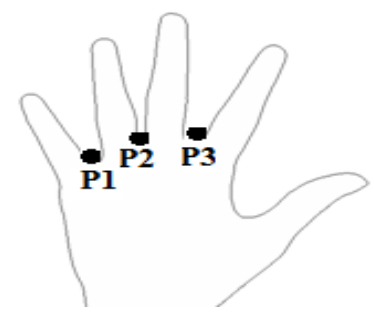

Figure 2 Key Points identified betwe en finger valley positio $n$

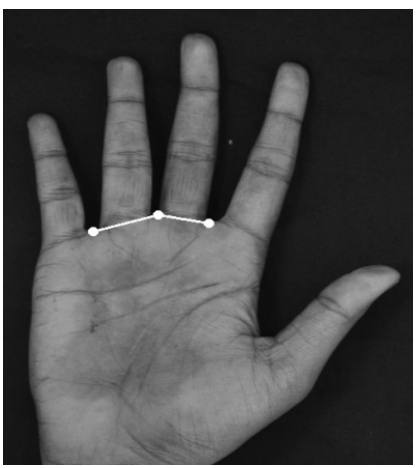

Figure 3 Hand image of first session

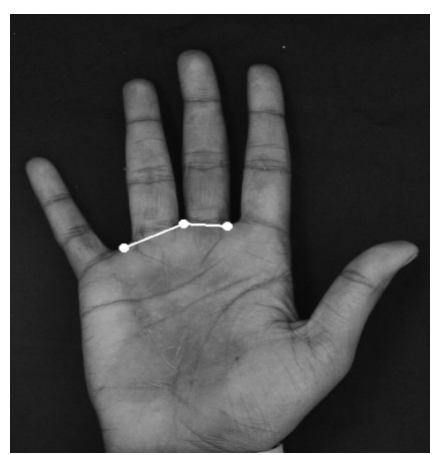

Figure 4 Hand image of second session

b. ROI of palm print is the rectangle region selected using the anchor points. The width of ROI is considered as the distance between anchor points and left top corner of rectangular region is selected 20 pixels just below the first anchor point.

The ROI part containing the palm print is cropped out of the main image and then resized to a size of 125 X 125 pixels. The resized palm print ROI image undergoes no further pre processing. A sample of the input image along with anchor points, palm print ROI region and extracted ROI of palm print is shown in Fig. 5 and 6 respectively.

Local binary pattern (LBP) has been widely used in biometric recognition systems to extract texture information from biometric images [24]. The LBP operator assigns a binary label to every pixel in the image by thresholding it against the eight neighbourhood pixels. If the pixel's value is 
greater than the neighbour a value 1 is assigned, otherwise 0 is assigned. A binary label is called uniform if it consists of at most two bit-wise transitions from 0 to 1 or vice-versa. For example 11101111 and 11111101 are uniform binary labels whereas 10101111 and 01011011 are nonuniform. There are 58 labels of uniform patterns and the rest 198 labels are of non-uniform. A label is given to each of the uniform patterns, and all other "non-uniform" patterns are assigned to a single label resulting in 59 labels. After all the labels have been determined, a histogram $\mathrm{H}_{l}$ of the labels is constructed as

$$
H_{l}=\sum\{L(i, j)=l\}, l=0, \ldots, \mathrm{n}-1
$$

Where $\mathrm{n}$ is the number of different labels produced by the LBP operator, while $\mathrm{i}$ and $\mathrm{j}$ refer to the pixel location. LBP histograms calculated from palm print ROI are stored as feature vectors of 59 dimensions.

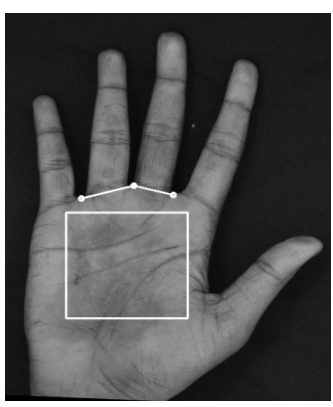

Figure 5 Identified palm ROI region

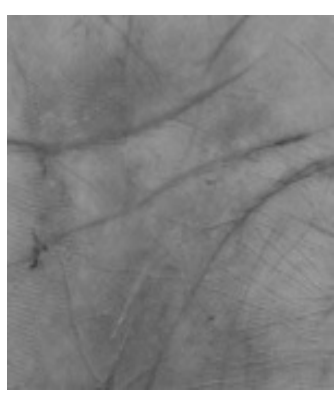

Figure 6 Extracted ROI of palm

\subsection{Hierarchical approach}

In our work we use angle feature as coarse level feature to partition the database into several bins. Although partitioning reduces the search space, comparing the query template sequentially with all the templates of a partition is still expensive in terms of search time. Traditional database are indexed numerically or alphabetically to reduce the retrieval time of records from database. In biometric database, biometric templates do not posses any natural or alphabetical order. Hence, there is a need of indexing technique which stores the templates in some predefined manner in such a way that an efficient retrieval strategy can be used. In our proposed approach we make use of a tree structure to organize the data. To facilitate retrieval strategy, we have used Kd tree [25] to implement the indexing operation on partitioned database.

kd-tree [26] is a binary tree that represents hierarchical sub division of space using splitting planes. It is a space partitioning data structure for organizing points in a $\mathrm{k}$ dimensional space and useful for searches involving multi dimensional search key. Number of nodes in a kd-tree is equal to number of templates in the gallery set and $\mathrm{k}$ denotes the dimensionality of the template. Each node in a kd-tree consists of information field, discriminator field, value field and two pointer fields. Information field contains descriptive information about the node, value field contains feature vector value, discriminator field will take a value from 1 to $\mathrm{k}$ (all nodes at any given level of a tree will have same discriminator value) and pointer fields will have pointer value of left $\&$ right sub tree of the node. At the root data points are split into two halves by a partition hyper plane and each half is assigned to one child node. Each of the two halves is recursively split in the same way to create a balanced binary tree. The proposed system reduces the search time since kdtree supports range search with good pruning. 


\subsubsection{Binning and Indexing}

Let $B=\left\{\left(b_{1 L}, b_{1 U}\right),\left(b_{2 L}, b_{2 U}\right), \ldots . .\left(b_{m L}, b_{m U}\right)\right\}$, be the bin class representative set. Where ( $\left.b_{i L}, b_{i U}\right)$ corresponds to interval values assigned to $i^{\text {th }}$ bin such that $b_{1 L}<b_{1 U}<b_{2 L}<b_{2 U}<\ldots . b_{m L}$ $<b_{\mathrm{mU}}$ and $\mathrm{m}$ represents number of bins. $b_{1 \mathrm{~L}}$ is the least value that angle feature can take and $\mathrm{b}_{\mathrm{mU}}$ is the maximum value.

The steps carried out in this phase are as follows.

a) An Hand image from gallery set is pre processed to locate key points.

b) Angle feature and palm print feature of hand image are computed as explained in feature extraction section to get feature vectors (templates). ID's of each template is also maintained.

c) Angle feature vector value is compared with bin interval values to find to which bin it maps to. Corresponding palm print feature vector is stored into the mapped bin.

d) Step (a) to (c) is repeated for all images in gallery set.

e) Form kd-trees for each bin using the palm print features stored in respective bins. ID's of templates mapped are also maintained.

\subsubsection{Identification Strategy}

Algorithm for retrieval of top best match for a query image is given below.

Algorithm Identification:

Step 1: For a query image q perform pre processing and feature extraction procedure to get the query angle feature vector and palm print feature vector.

Step 2: Compare query angle value with bin interval values to find to which bin it maps.

Step 3: A ' $k$ ' nearest neighbour search on Kd-tree of selected bins is invoked using the palm print feature vector. These nearest neighbours form the candidate set $C$ that contains identities of templates satisfying $\forall \mathrm{i} \in \mathrm{C}$

$$
\|q-i\| \leq\|q-p\|, \quad \forall \mathrm{p} \in\left(\mathrm{DB}_{\mathrm{G}}-\mathrm{C}\right)
$$

Where $\|$. $\|$ is distance measure.

Step 4: Accumulate only those identities in $\mathrm{C}$ whose distance is less than threshold $\mathrm{T}$.

Step 5: Declare the top best match as the identity of q.

\section{EXPERIMENTAL RESULTS}

To evaluate the performance of proposed approach four images of each individual from first session are considered for gallery set resulting in 1792 images in gallery set. Any one image out of four images from second session is randomly selected for probe set resulting 448 images in probe set. In our work we use Euclidean distance between a probe template and a gallery set template for describing the similarity between them. A threshold is used to regulate the system decision. If the Euclidean distance between the pair of templates computed is less than the threshold then the pair of templates is considered to belong to the same person. Consequently if the distance is greater than the threshold then the pair of templates is considered to belong to different person. Performance of the proposed scheme is evaluated using three measures namely Bin penetration rate, Correct Index Power (CIP) and Bin miss rate. A query image is said to be correctly identified, if one of the retrieved identities corresponds to the correct identity.

The Bin penetration rate $\operatorname{Pr}$ is defined by 


$$
P_{r}=\frac{N_{S} \times A_{T}+N_{B}}{N_{D}}
$$

where $N_{S}$ is the number of bins to be searched, $A_{T}$ is the average templates per bin, $N_{B}$ is number of bins and $N_{D}$ is the size of the database.

CIP is defined by

$$
C I P=\frac{C}{P}
$$

Where $\mathrm{C}$ is the number of query images correctly identified and $\mathrm{P}$ is the number of query images in the probe set.

Bin miss rate is defined by

$$
B_{r}=1-\frac{B}{L}
$$

where B is the number of times a query's corresponding identity is found in the selected bin and L is the total number of queries.

Hand geometry features of gallery set images are used to find the ID's that are mapped into bins. In our work we have selected the number of bins as six and this value is based on initial experiments carried out to find the minimum and maximum angle value of gallery set images. The efficiency of the binning scheme is measured in terms of bin miss rate and bin penetration rate. Figure 7 shows the effect of varying number of bins on bin penetration rate. Figure 8 shows the relationship between bin miss rate and number of bins selected. From figure 8 we can infer that bin miss rate is zero if three closet bins are selected.

Figure 9 shows the relationship between bin miss rate and bin penetration rate with respect to number of bins selected. The number of bins selected is considered as optimal value where the two curves intersect. Since the two curves intersect for a $x$-axis value between 2 and 3, number of bins selected for identifying probe is considered as 3 . This analysis is done assuming a uniform distribution of bin density for all the bins. The actual bin density are 260, 290, 330,310,352,250. If probe template maps to first bin or second bin, then bin 1,2 and 3 are selected. If mapped to third bin then bin 2,3 and 4 are selected and for mapping to fourth and fifth bin then bin 4,5 and 6 are selected. Our analysis of penetration rate hold true since when three consecutive bins are selected average percentage of gallery set templates considered is almost equal $52 \%$. This fact is illustrated in table 1. In conventional approach a query template is compared with all 1792 templates $(100 \%)$ stored in the database. With the proposed approach percentage of database considered for second stage is $52 \%$, hence database filtered at first stage by binning approach is $48 \%$. 
International Journal on Computational Science \& Applications (IJCSA) Vol.5, No.5,October 2015

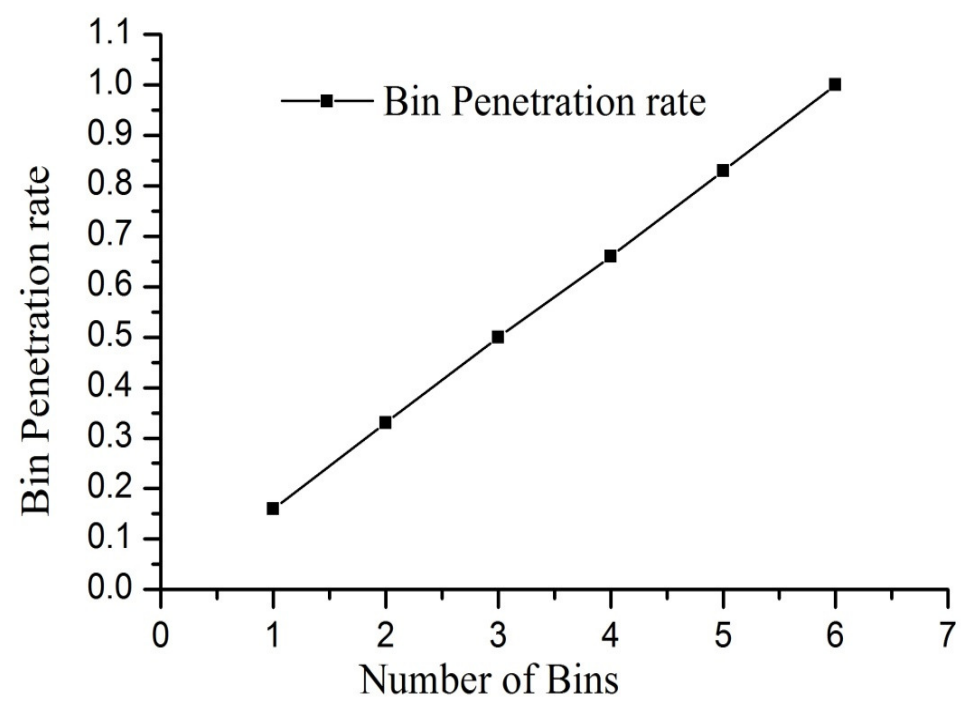

Figure 7 Relationship between Bin penetration rate and Number of bins

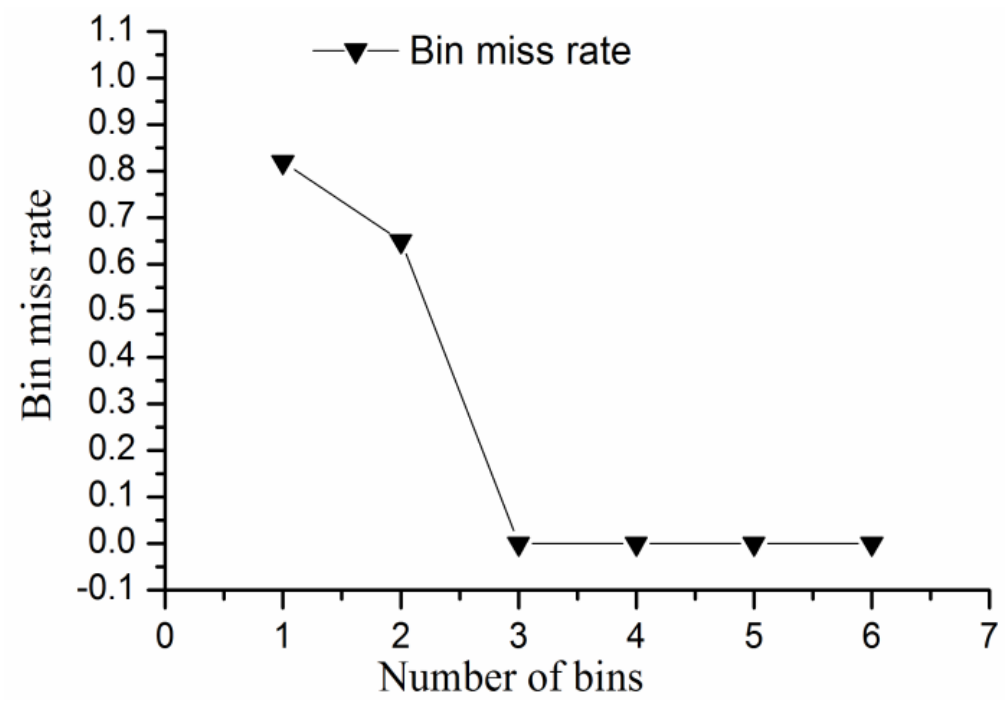

Figure 8 Relationship between Bin miss rate and Number of bins 


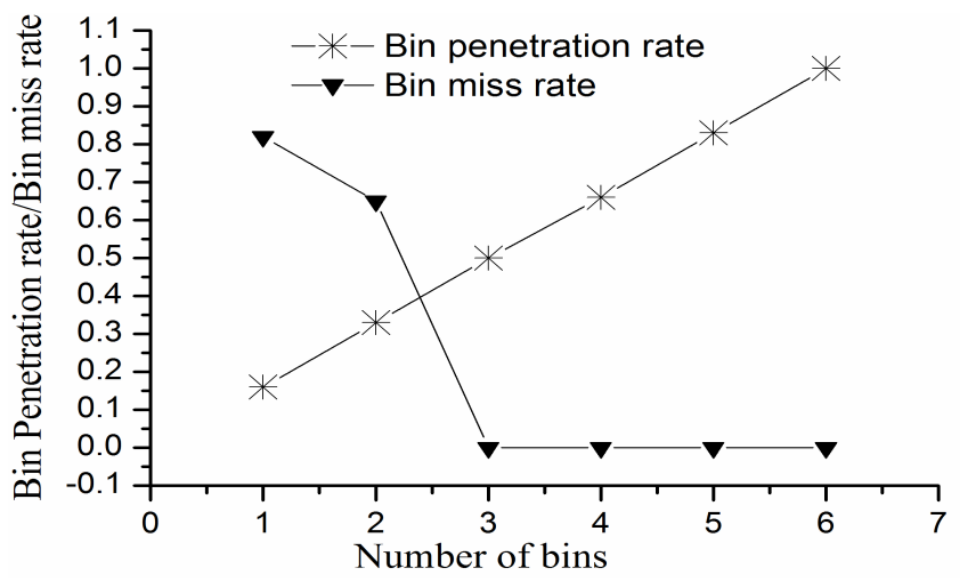

Figure 9 Relationship between Bin miss rate/ Bin penetration rate and Number of bins

In order to fix the threshold value verification experiments were carried out for palm print modality using LBP features. Personal verification is one to one matching. Verification refers to confirming or denying a person's claimed identity. Two types of error rates namely false rejection rate (FRR) and false acceptance rate (FAR) are defined in biometric literature with respect to verification method. FRR is the percentage of authorized users that the biometric system fails to accept and FAR is the percentage of unauthorized users that the biometric system fails to reject. Equal error rate (ERR) is the optimal rate when FAR equals FRR. The threshold value obtained when FAR equals FRR is 0.0005 . This threshold value is used in steps 4 of identification algorithm.

Nearest neighbour(NN) search on the selected three kd-trees is invoked for each query palm print template selected from probe set as mentioned in identification algorithm to get candidate lists. Since k-NN search uses Euclidean distance, from the three kd-trees all identities with distance less than threshold $\mathrm{T}$ are considered for candidate list. Also $\mathrm{k}$ value is varied from $10 \%$ of entries in kd-tree to $100 \%$.

Table 1 Penetration rate analysis with proposed approach and conventional approach

\begin{tabular}{|l|c|c|c|c|c|c|c|}
\hline & \multicolumn{5}{|c|}{ With proposed approach } & Conventional \\
\cline { 2 - 8 } & Bin 1 & Bin 2 & Bin 3 & Bin 4 & Bin 5 & Bin 6 & $\begin{array}{c}\text { Complete gallery } \\
\text { set }\end{array}$ \\
\hline $\begin{array}{l}\text { Number of } \\
\text { templates in }\end{array}$ & 260 & 290 & 330 & 310 & 352 & 250 & 1792 \\
\hline $\begin{array}{l}\text { Number of } \\
\text { templates } \\
\text { considered } \\
\text { when probe } \\
\text { template is } \\
\text { mapped to }\end{array}$ & 880 & 880 & 930 & 992 & 912 & 912 & 1792 \\
\hline $\begin{array}{l}\text { Penetration } \\
\text { rate }\end{array}$ & $49 \%$ & $49 \%$ & $52 \%$ & $55 \%$ & $51 \%$ & $51 \%$ & $100 \%$ \\
\hline
\end{tabular}




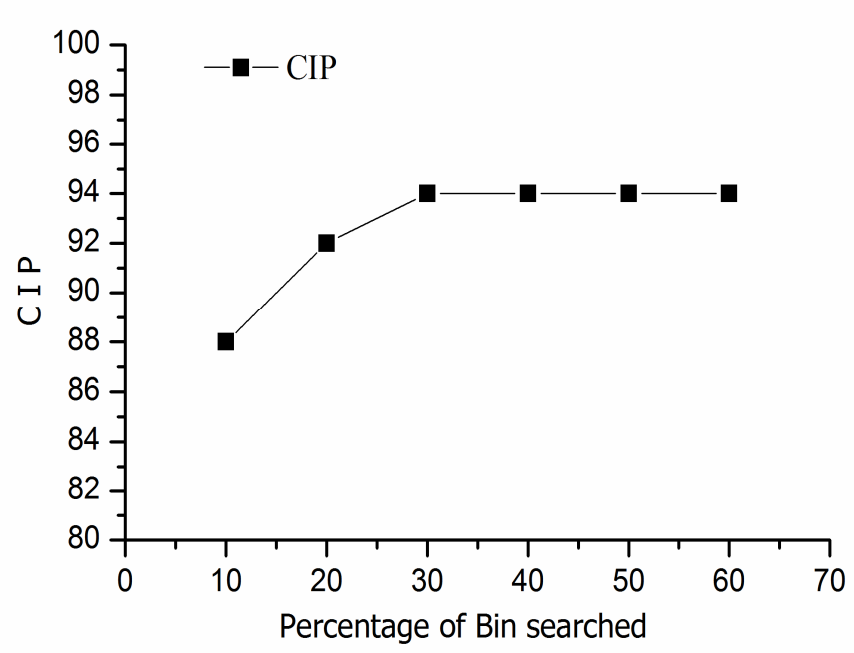

Figure 10 Plot of CIP for varying percentage of Bin search

The CIP curve obtained for varying $\mathrm{k}$ value is shown in figure 10. From this plot we can infer that corresponding identities are found by searching $30 \%$ of nearest neighbours in kd-tree. It can be noted that $94 \%$ identification accuracy is achieved and this is the same accuracy that is achieved by sequential approach.

Biometric system proposed in [27-30] makes use of more than one modality to enhance the system performance. These approaches establish the identity associated with query image by considering the complete gallery set and focuses mainly on enhancing the accuracy. However proposed work focuses on reducing response time by reducing search space while maintaining accuracy. Also majority of research work uses palm print images acquired using pegs or holdings where as in our proposed work contactless image acquisition set up is used.In conventional approach, test template corresponding to a test image selected from probe set is compared with templates of all images from the gallery set. This exhaustive comparison can make the response time of system very poor due to more number of comparisons.The computational time analysis of proposed approach and conventional approach methods for searching one query template of probe set against gallery set templates is tabulated in Table 2. From Table 2 it is clear that the proposed approach is more effective than the conventional approach. Time for preprocessing and ROI extraction of one hand image is $2 \mathrm{sec}$ and time for feature extraction is $0.125 \mathrm{sec}$.

Table 2 Time analysis of proposed approach and conventional approach

\begin{tabular}{|l|l|l|l|l|l|}
\hline & \multicolumn{4}{|l|}{ With proposed approach } & Conventional \\
\hline$\%$ of bin searched & $10 \%$ & $20 \%$ & $30 \%$ & $40 \%$ & $100 \%$ \\
\hline $\begin{array}{l}\text { Time for estblaishing the } \\
\text { identity using probe } \\
\text { feature vector }\end{array}$ & $0.005 \mathrm{sec}$ & $0.007 \mathrm{sec}$ & $0.01 \mathrm{sec}$ & $0.015 \mathrm{sec}$ & $0.96 \mathrm{sec}$ \\
\hline $\begin{array}{l}\text { Time for overall process } \\
\text { for one probe image }\end{array}$ & $2.13 \mathrm{sec}$ & $2.132 \mathrm{sec}$ & $2.135 \mathrm{sec}$ & $2.14 \mathrm{sec}$ & $3.085 \mathrm{sec}$ \\
\hline Correct index power in \% & 88 & 92 & 94 & 94 & 94 \\
\hline
\end{tabular}




\section{Conclusions}

An novel binning and indexing approach to retrieve identity of a query image based on hand geometry and palm print features is proposed in this paper. For a query feature template using hand geometry feature three kd trees are selected in the first stage by binning approach. Since kdtree is used to index the partitioned database a nearest neighbor search is invoked on the selected $\mathrm{kd}$-trees to retrieve the top matches. These top matches are subsequently used for person identification. The obtained experimental results are encouraging and we claim that proposed binning and indexing approach gives the same identification accuracy as obtained by conventional database search with 0.95 seconds reduction in search time for $48 \%$ reduction in search space.

\section{REFERENCES}

[1] Anil K .Jain ,"An Introduction to Biometric Recognition”, IEEE transactions on circuits and systems for video technology, vol. 14, no. 1, pp. 1-20,January 2004.

[[2] A.Ross,AnilK.Jain,"Multimodal biometrics: an overview”, Proceedings of 12th European Signal Processing Conference, Poland, pp 1121-1124, September 2007.

[3] Kresimir Delac,Mislav Grgic, “ A survey of biometric recognition methods",46th International Symposium Electronics in Marine, pp 184-193,June 2004.

[4] X. Wu, K. Wang and D. Zhang, "Line feature extraction and matching in palmprint", in Proceeding of the Second International Conference on Image and Graphics, pp. 583-590, 2002

[5] X. Wu, K. Wang and D. Zhang, "A novel approach of palm-line extraction", in Proceeding of the Third International Conference on Image and Graphics, pp 230-233, 2004.

[6] R.K. Rowe, U. Uludag, M. Demirkus, S. Parthasaradhi and A.K. Jain, "A multispectral whole-hand biometric authentication system", Proceedings of Biometric Symposium Biometric Consortium Conference, Baltimore, September, 2007.

[7] T. Connie, T. Andrew, K. Goh, "An automated palmprint recognition system", Image and Vision Computing, vol. 23, 2005, pp. 501-505.

[8] X. Wu, D. Zhang, K. Wang,, "A Fisherpalms based palm-print recognition",Pattern Recognition Letters, vol. 24, 2003, pp. 2829-2838.

[9] D. Zhang, W.K. Kong, J. You and M. Wong, "On-line palmprint identification", IEEE Transactions on Pattern Analysis and Machine Intelligence, vol. 25, no. 9, pp. 1041- 1050, 2003.

[10] C.C. Han, "A hand-based personal authentication using a coarse-to-fine strategy", Image and Vision Computing, vol. 22, no. 11, pp. 909-918, 2004.

[11] L. Shang, D.S. Huang, J.X. Du and Z.K. Huang, "Palm-print recognition using ICA based on winnertake-all network and radial basis probabilistic neural network", LNCS 3972,pp. 216-221, 2006.

[12] E. Ardabili, K. Maghooli, E. Fatemizadeh, "Contourlet Features Extraction and AdaBoost Classification for Palmprint Verification ", Journal of American Science 7(7):353-362(ISSN: 15451003),2011

[13] Deepti Tamrakar, Pritee Khanna, "Analysis of Palmprint Verification using Wavelet Filter and Competitive Code", In-ternational Conference on Computational Intelligence and Communication Networks, 2010.

[14] W. Li, D. Zhang, Z. Xu, "Palmprint identification by Fourier transform", International Journal of Pattern Recognition and Artificial Intelligence, vol. 16, no. 4, pp. 417-432,2002.

[15] G. Lu, K. Wang and D. Zhang "Wavelet based feature extraction for palmprint identification", In Proceeding of Second International Conference on Image and Graphics, pp. 780-784, 2002.

[16] X. Wu, D. Zhang, K. Wang and B. Huang,"Palmprint classification using principle lines", Pattern Recognition, Vol 37, No 10, pp1987-1998, Oct 2004

[17] W. Jia, D.S Huang and D. Zhang, "Palmprint verification based on robust line orientation code", Pattern Recognition, vol. 41, no. 5, pp. 1504-1513, 2008

[18] X. Wu, D. Zhang, K. Wang, "A Fisherpalms based palm-print recognition", Pattern Recognition Letters, vol. 24, 2003, pp. 2829-2838. 
[19] Rahman, A., Anwar, F. \& Azad, S.," A simple and effective technique for human verification with hand geometry", Computer and Communication Engineering, 2008.ICCCE 2008. International Conference on, pp. 1177-1180.

[20] Kumar, A.;Wong, D.; Shen, H.; Jain, "A. Personal Verification using Palmprint and Hand Geometry Biometrics", In Proceedings of the 4th International Conference on Audio- And Video-Based Biometric Personal Authentication, Guildford, UK, 9-11 June 2003.

[21] Vivek Kanhangad, Ajay Kumar and David Zhang, "Contactless and Pose Invariant Biometric Identification Using Hand Surface", IEEE Transactions on Image Processing, Vol. 20, No. 5, May 2011, pp.1415-1424.

[22] A. Kumar and D. Zhang, "Hand geometry recognition using entropy based discretization", IEEE Transactions. Information Forensics Security, vol. 2, no. 2, pp. 181-187, Jun. 2007.

[23] G.Michael, T. Connie,T Andrew, “Touchless palm print biometrics:Novel design and implementation”, Image and vision computing, Vol 26, Issue 12,pp 1551-1560, 2008.

[24] T. Ojala, M. Pietikainen and T. Maenpaa, "Multiresolution gray-scale and rotation invariant texture classification with local binary patterns", IEEE transaction on Pattern Analysis and Machine Intelligence (PA MI), vol. 27(7), pp 971-987, 2002.

[25] M L Anitha and K A RadhaKrishna Rao, " An Efficient Palmprint Identification system based on an Indexing approach", International Conference on Advances in Computing, Communications and Informatics (ICACCI), SJCE,Mysore, pp688-693, 2013.

[26] You Jia ,Jing dong Wang,,Gang Zeng, Hongbin Zha, Xian-Sheng Hua, “Optimizing kd trees for scalable visual descriptor indexing", CVPR, pp3392-3399, 2010.

[27] M.P.Dale, M.A,Joshi, H.J.Galiyawala,"A single Sensor Hand Geometry and Palm Texture Fusion for person identification", International journal of Computr Applications, Vol 42, No 7, 2013.

[28] Aditya Nigam. A. Palguni Gupta, "Multilmodal Personal Authentication system Fusing Palm print and knuckle print', In: Huang, D.S.,et al. (eds.) ICIC 2013. CCIS 375, pp 188--193, Springer, Heidelberg 2013.

[29] G.Michael, T. Connie, T,Andrew, "An innovative contactless palm print and knuckle print recognition system", Pattern Recognition letters, Issue 31 ,pp 1708-1719, 2010.

[30] Ferrer M A, Morales A, Travieso C.M, Alonso J.B, "combining hand biometric traits for personal identification", 43rd Annual 2009 International Carnahan Conference on Security Technology, pp 155 - 159 ,oct 2009. 